Fig. 1 Distribution of pain scores during the intravitreal injection procedure under topical anaesthesia, according to the patient's appreciation
Number of patients vs Score of pain

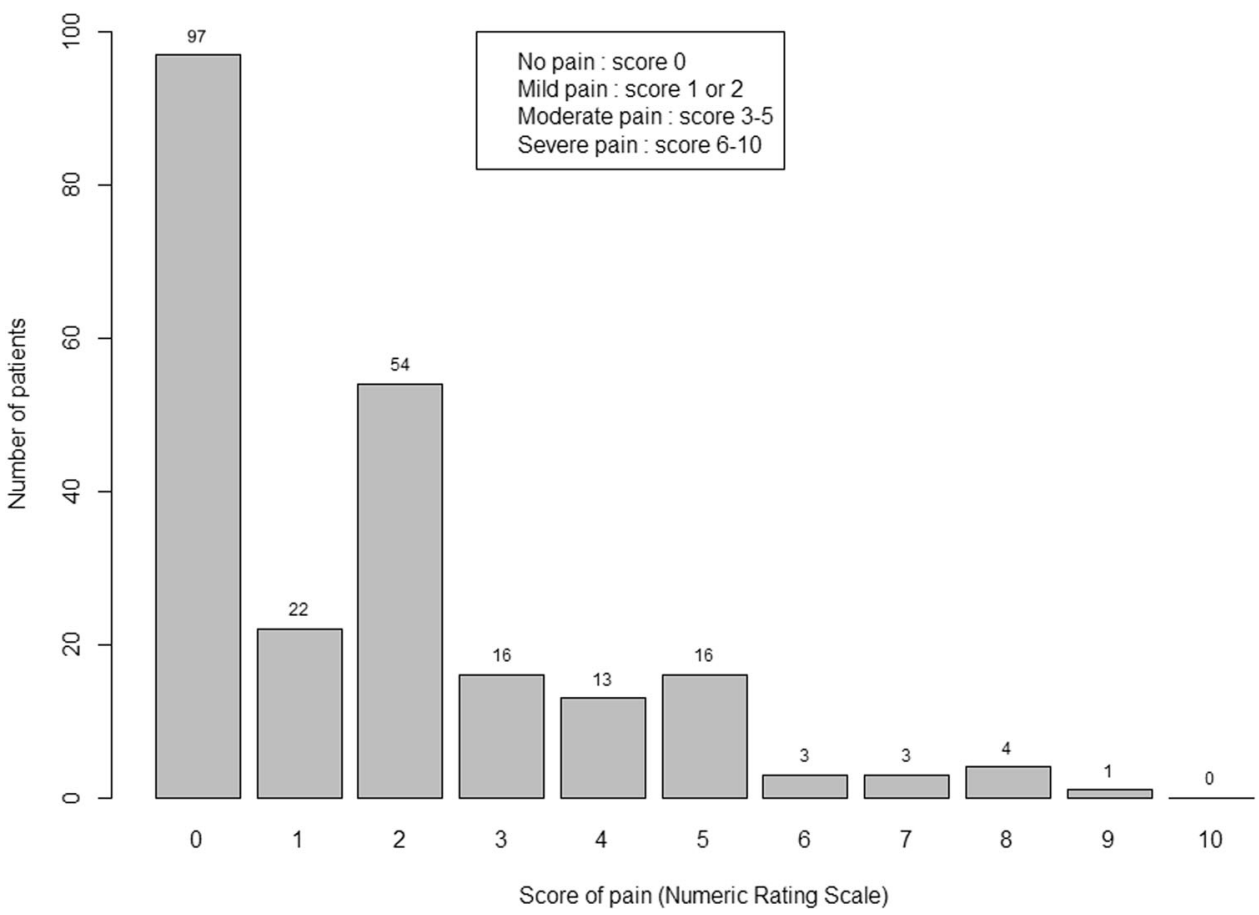

\section{References}

1. Rifkin L, Schaal S. Factors affecting patients' pain intensity during in office intravitreal injection procedure. Retina. 2012;32:696-700.

2. Segal O, Segal-Trivitz Y, Nemet AY, Cohen P, Geffen N, Mimouni M. Anxiety levels and perceived pain intensity during intravitreal injections. Acta Ophthalmol. 2016;94:203-4.

3. Okamura H, Fukui S, Nagasaka Y, Koike M, Uchitomi Y. Psychoeducational intervention for patients with primary breast cancer and patient satisfaction with information: an exploratory analysis. Breast Cancer Res Treat. 2003;80:331-8.

4. Nekolaichuk C, Watanabe S, Beaumont C. The Edmonton Symptom Assessment System: a 15-year retrospective review of validation studies (1991--2006). Palliat Med. 2008;22:111-22.

5. van der Heijden MJ, Oliai Araghi S, van Dijk M, Jeekel J, Hunink MG. The effects of perioperative music interventions in pediatric surgery: a systematic review and meta-analysis of randomized controlled trials. PLoS ONE. 2015;10:e133608.

\title{
Management of anterior capsular contraction syndrome: pitfall of circular capsulotomy technique with the neodymium YAG laser
}

\author{
Craig Wilde $^{1} \cdot$ Andrew Ross $^{1} \cdot$ Mary Awad ${ }^{1} \cdot$ Hean-Choon Chen ${ }^{2} \cdot$ Harminder S Dua $\mathbb{D}^{1}$
}

Received: 7 March 2018 / Accepted: 15 March 2018 / Published online: 18 May 2018

(c) The Royal College of Ophthalmologists 2018

\section{Harminder S Dua}

Harminder.Dua@nottingham.ac.uk

1 Ophthalmology and Vision Sciences, Division of Clinical Neurosciences, B Floor, EENT Centre, Queen's Medical Centre, University of Nottingham, Nottingham, UK

2 Royal Derby Hospital, Uttoxeter Road, Derby DE22 3NE, UK
Dear Editor,

Capsular contraction syndrome (CCS) is a distinct clinical entity associated with continuous curvilinear lcapsulorhexis (CCC) performed during phacoemulsificaion [1]. Although some degree of anterior capsular whitening and 
Fig. 1 a Mobile capsular fragment in the inferior $\mathrm{AC}$ of case 2 . The cornea was clear and there was no anterior chamber activity. b Anterior segment optical coherence tomogram of case 1 showing adhesion of the complete doughnut shaped capsular remnant to the corneal endothelium. c Diffuse slit lamp view of capsular fragment (arrows). d Post-YAG laser anterior capsulotomy showing the cut edge of the anterior capsule in a complete circular pattern, highlighted with a dashed line. The radial white lines illustrate the direction of the recommended radial cuts, perpendicular to the edge of the capsule margin
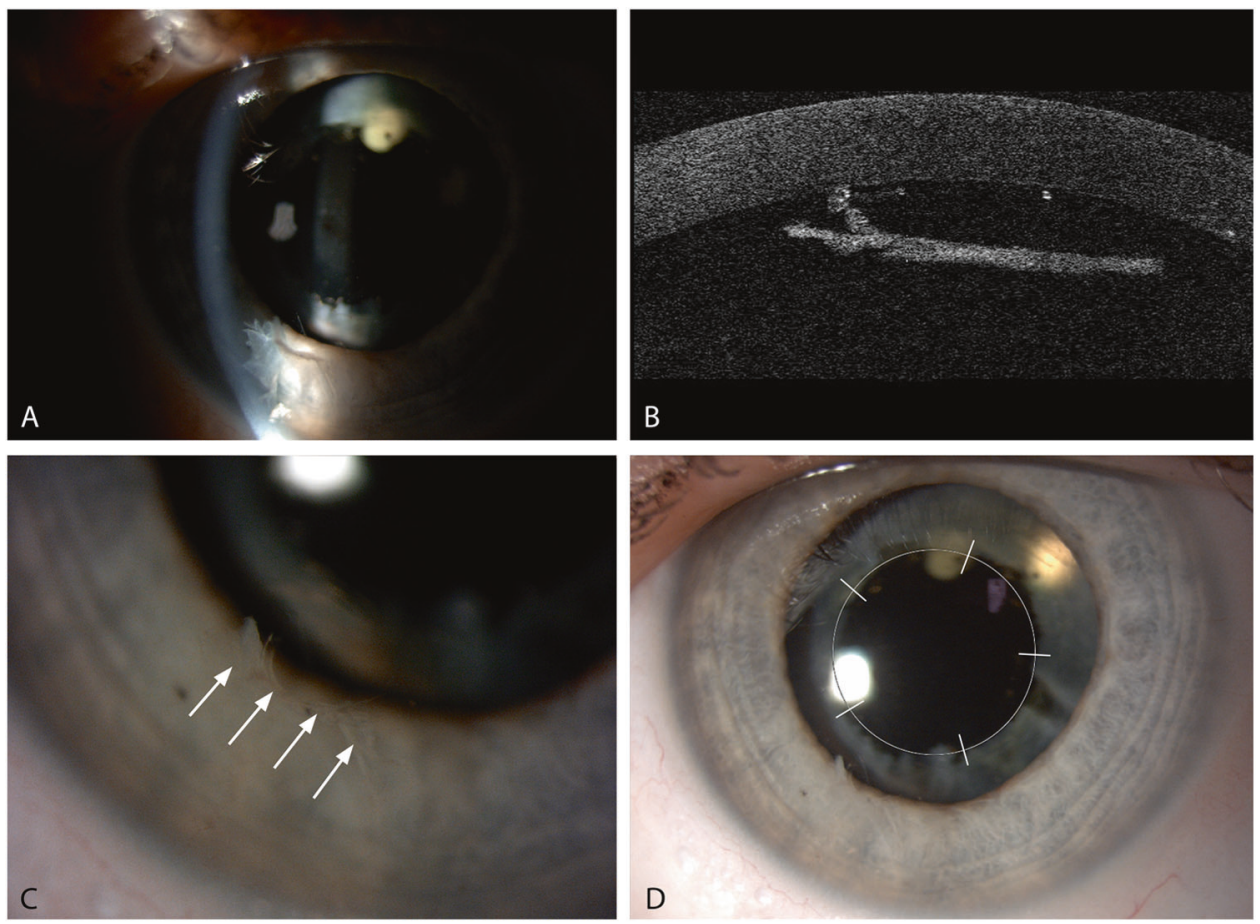

fibrosis is normal, extreme phimosis can become significant when it leads to visual axis opacification, secondary tilting, decentration, buckling, or posterior displacement of the intraocular lens (IOL) with hypermetropic shift [1]. The clinical impact is more with toric and multifocal lens implants. Extreme complications, such as lens dislocation, retinal, and ciliary body detachments can also occur [1].

The primary procedure employed to manage CCS is the use of Nd: YAG laser to create several radial incisions around the capsulorhexis margin [2], either in a linear or in a parabolic fashion. This releases the tension created by the phimosis, allowing the optic to return to its original position. Surgical approaches are numerous and include the use of a vitrector to create a circular capsular opening and removal of capsular and fibrotic remnants [3], along with intraocular diathermy [3] and incisions with microscissors [4]. A technique using straight scissors to cut the anterior capsule and subsequent completion of a CCC at the edge of the optic with forceps, using the force of adhesion between the anterior and posterior capsule has also been described [4].

Here we report two cases where anterior YAG laser capsulotomy was performed in a continuous circular fashion. The incised doughnut shaped fragment dropped into the anterior chamber (AC) (Fig. 1a). The first case was a woman aged 81 with posterior capsular opacification (PCO) and opacification of the anterior capsule with phimosis several years post phacoemulsification. She also had an idiopathic macular hole for which the capsulorhexis was enlarged to enhance the view during vitrectomy. The peripheral margin of the displaced remnant attached to the inferior corneal endothelium (Fig. 1b) and was fixed.

The second case was a 26-year-old female with a previous history of proliferative diabetic retinopathy treated with vitrectomy, endolaser and silicone oil fill. Subsequent cataract surgery combined with silicone oil removal resulted in CCS. YAG laser capsulotomy was performed in a circular fashion, creating a free-floating fragment (Fig. 1c). This settled in the inferior AC but changed position with head posture, obscuring vision during reading.

Both cases highlight problems of performing circular anterior capsulotomies. Free-floating remnants can be large and mobile, directly interfering with vision and pose risk of endothelial cell loss with associated corneal edema [5]. Their presence may necessitate surgical removal. Although there are no case series addressing risk of retained capsular remnants within the $\mathrm{AC}$, retained nuclear fragments pose substantial risk of corneal edema making their removal essential. The authors recommend YAG anterior capsulotomy in a spoke like pattern, radiating perpendicularly from the edge of the capsule margin, as illustrated (Fig. 1d). We recommend at least 4 initial cuts of $1 \mathrm{~mm}$, which can be extended further as required depending on the severity of the CCS and eccentricity of the capsule/intraocular lens complex. The cuts should generally not extend beyond the edge of the optic.

This method is safe and effective [2]. The effect is sometimes not instantaneous, but if this technique fails, further radial YAG laser capsulotomy or surgical approaches can be considered. Given the low incidence of CCS, 
training opportunities in its management are limited. Trainees should be aware of the different methods and their limitations.

\section{Compliance with ethical standards}

Conflict of interest The authors declare that they have no conflict of interest.

\section{References}

1. Davison JA. Capsule contraction syndrome. J Cataract Refract Surg. 1993;19:582-9.
2. Tsinopoulos I, Symeonidis C, Frangou E, Dimitrakos SA. Capsule contraction syndrome in eight cases of hydrophobic one-piece intraocular lens implantation. Clin Exp Optom. 2008;91:469-72.

3. Yeh PC, Goins KM, Lai WW. Managing anterior capsule contraction by mechanical widening with vitrector-cut capsulotomy. J Cataract Refract Surg. 2002;28:217-20.

4. Panagopoulos A, Chalioulias K, Kirkby GR. A new approach in the surgical management of anterior capsular phimosis syndrome. Ophthalmic Res. 2009;42:221-3.

5. Wilson RP, Gupta R. Focal corneal decompensation caused by an anterior capsulotomy remnant. J Cataract Refract Surg. 1997;23: $1273-4$.

\title{
Comment on: Assessment of patient-reported outcome and quality of life improvement following surgery for epiphora
}

\author{
Valerie Juniat $^{1} \cdot$ Sarju Athwal ${ }^{1} \cdot$ Adeela Malik $^{2} \cdot$ Mona Khandwala $^{1}$ \\ Received: 11 January 2018 / Accepted: 12 February 2018 / Published online: 18 May 2018 \\ (c) The Royal College of Ophthalmologists 2018
}

\section{Dear Editor,}

We read with great interest the article by Sipkova et al. [1] entitled "Assessment of patient-reported outcome and quality of life improvement following surgery for epiphora" in the December 2017 issue of Eye. We have reviewed the patient-reported outcomes of our own patients who have undergone surgery for epiphora. We would like to share our experience with readers.

Sipkova et al. [1] discussed the relevant limitations of the Glasgow Benefit Inventory (GBI), Munk symptoms score [2], and Lac-Q questionnaire. In our study, we prospectively enrolled 17 patients (20 eyes) who underwent lateral tarsal strip procedures for epiphora and lower lid laxity between June 2014 and September 2015. All 17 patients (20 eyes) completed the Munk score preoperatively and at 3 months

Valerie Juniat

vjuniat@doctors.org.uk

1 Maidstone and Tunbridge Wells NHS Trust, Hermitage Lane, Maidstone, Kent ME16 9QQ, UK

2 Moorfields Eye Hospital NHS Trust, 162 City Road, London EC1V 2PD, UK postoperatively. Patients subjectively scored their epiphora severity based on the $0-4$ scale, where. is no watering and. is constant watering. Statistical analysis was carried out using paired $T$-test. Average Munk score prior to lid surgery was $4.65 \pm 0.49 \mathrm{SD} \quad($ median $=5)$ and $2.05 \pm 1.05 \mathrm{SD}$ (median $=2$ ) postoperatively. The mean difference in preoperative and postoperative Munk score was $2.6 \pm 1.05 \mathrm{SD}$ (median $=3, p<0.001,95 \%$ CI 2.11-3.09). Only one patient had no change in their Munk score following lateral tarsal strip surgery, scoring. both pre and postoperatively.

Our study findings compare with those from Sipkova et al. [1], where their patients also showed significant improvement in Munk score following lid-tightening surgery $(p<0.001)$. In our experience, the Munk score can be applied realistically during busy outpatient clinical settings in view of its ease of completion. However, the Munk score has its limitations in assessing the impact of epiphora on specific daily tasks. For example, our single patient who did not show improvement in Munk score may have had very severe frequency of wiping from 50 times. day preoperatively (Munk score of 4) to 20 times. day postoperatively (Munk score of 4), an improvement for the patient. We therefore support Sipkova et al. [1] in their 